\title{
$\operatorname{arCOS}$ DESIGN
}

ARCOS Design é uma publicação semestral

Programa de Pós-Graduação em Design

Escola Superior de Desenho Industrial

Universidade do Estado do Rio de Janeiro

Rua Evaristo da Veiga, 95, Centro

Rio de Janeiro, RJ 20031-040

Tel: $2232-6908$ | 6909 | 6910

e-mail: revistaarcos@gmail.com

ISSN: 1984-5596

Rio de Janeiro, V.9 N. 2, Dezembro 2016.

http://www.e-publicacoes.uerj.br/index.php/arcosdesign

\section{Editores Principais}

Barbara Szaniecki, DSc - PPD ESDI/UERJ

Jorge Lúcio, DSc - PPD ESDI/UERJ

Sydney Freitas, DSc - PPD ESDI/UERJ

\section{Conselho Editorial}

André Monat, DSC - PPD ESDI/UERJ

João de Souza Leite , DSc - PPD ESDI/UERJ

Lucy Niemeyer, DSc - PPD ESDI/UERJ

Washington Dias Lessa, DSc - PPD ESDI/UERJ

\section{Editores Executivos}

Camille Moraes - PPD ESDI/UERJ

Liana Ventura - PPD ESDI/UERJ

Philippe Leon Anastassakis - PPD ESDI/UERJ

Talita Tibola - PPD ESDI/UERJ 


\section{$\operatorname{arCOS} D E S I G N$}

\section{Avaliadores}

Alfredo Jefferson de Oliveira - PUC-Rio

André Fabio Villas-Boas - PUC-Rio

André Monat - ESDI/UERJ

Ari Antônio da Rocha - UFRN

Carla Galvão Spinillo - UFPR

Claudia Mont'Alvão - PUC-Rio

Cecilia Consolo - FACAMP e Lab Cognitivo

Cleomar de Souza Rocha - UNIFACS

Denise Portinari - PUC-Rio

Edna Cunha Lima - PUC-Rio

Eduardo Corte Real - IADE Lisboa, Portugal

Fernando Reiszel Pereira - ESDI/UERJ

Georg-Christof Bertsch, PhD - HFG-Offenbach, Alemanha

Guilherme Cunha Lima - ESDI/UERJ

Isabella Perrotta - ESPM

Jairo José Drummond Câmara - UEMG

João Carlos Lutz Barbosa - UFF

Jorge Lúcio de Campos - ESDI/UERJ

José Carlos Plácido da Silva - FAAC/UNESP

João de Souza Leite - ESDI/UERJ

Klaus Krippendorff, PhD - University of Illinois, Urbana, EUA

Laura Bezerra Martins - UFPE

Lia Buraque de Macedo - UFRGS

Lucy Niemeyer - ESDI/UERJ

Luis Carlos Paschoarelli - FAAC/UNESP

Marcos da Costa Braga - USP

Maria Cristina Volpi Nacif - EBA/UFRJ

Mônica Moura - USP

Priscila Lena Farias - USP

Reinhart Butter - Ohio State University, EUA

Stephania Padovani - UFPR

Susi Mariño Pequini - UNEB

Sydney Freitas - ESDI/UERJ

Thais Leticia Pinto Vieira - Unicarioca

Vicente Cerqueira - ESDI/UERJ

Victor Margolin - University of Illinois at Chicago (UIC), EUA

Washington Dias Lessa - ESDI/UERJ 


\section{$\operatorname{arCOS} D E S I G N$}

\section{Sumário da edição especial \\ Seminário Design.com \\ Outubro 2017}

Articulações

1-19

Queerizar o design

Denise Portinari

20-31 GreenUP HUB: projetando e construindo infraestruturas verdes funcionais no Brasil

Giacomo Pirazzoli

\section{Conexões}

32-38 Fazendo conexões: considerações preliminares sobre design e ação social

Daniel Portugal

39-54 Instrução para simbiose

Carolina Correia dos Santos

55-75 Design e a construção de sentidos sobre questões de interesse público

Clorisval Pereira Jr.

76-88 Ou duas ou três palavras sobre a palavra design ou Frederico Guilherme Bandeira de Araujo, Heitor Levy Ferreira Praça e Letícia Castilhos Coelho

\section{Expansões}

89-101 Urbanismo tático e intervenções urbanas: aderências e deslizamentos

Pedro Caetano Eboli Nogueira 


\title{
$\operatorname{arCOS} D E S I G N$
}

\section{Sumário da edição especial \\ Seminário Design.com \\ Outubro 2017}

\author{
(Continuação)
}

102-126 Urbanismo crítico e design na perspectiva de uma cartografia da ação dos subalternos

Pedro Cláudio Cunca Bocayuva

127-146 Como a Arquitetura + Design + Urbanismo contribuem no / para o processo de (re)invenção de identidades das cidades? Ana Beatriz da Rocha

147-162 “Rio Comprido em Nós": do espaço público à construção do público por meio do design

Mariana Costard

163-179 Mapa-Praça-Máquina: experimentos de design no espaço público

Barbara Szaniecki, Liana Ventura, Philippe Leon Anastassakis e Talita Tibola 


\section{$\operatorname{arCOS} D E S I G N$}

\section{Apresentação da edição especial Seminário Design.com Outubro 2017}

A segunda edição do seminário DESIGN.COM aconteceu na Escola Superior de Desenho Industrial (ESDI/UERJ) de 30 de maio a 01 de junho de 2017 às 17h. Foram três dias de debate sobre as articulações do design com outros saberes e fazeres, sobre as expansões do design pela cidade entendida ela mesma como um espaço-tempo com dimensão real e virtual e, por fim, sobre suas conexões com outros atores.

As articulações do design podem constituir algo como uma ecologia de saberes e fazeres.. Em tempos de urgências locais e globais, o design tem se articulado a várias disciplinas de modo a criar possibilidades de produzir, a partir da criatividade e imaginação, vidas mais saudáveis e sustentáveis. Partindo da proposição de uma ecologia de práticas - que não separe de maneira estanque as disciplinas, tampouco o humano de seu meio ambiente, essas questões foram abordadas tomando o design não apenas como um articulador entre diversos campos de conhecimento, mas como abordagem de problemas de maneira complexa entre diferentes atores e subjetividades, uma ecologia de práticas que produz processos plurais e colaborativos entre humanos e não humanos. Ao tomar a ecologia como um modo amplo de pensar, pode o design produzir convergências entre saberes e fazeres, novas comunicações entre diferentes poderes e novas convivências entre seres? Desta mesa participaram Denise Portinari (PPG-Design / PUC-Rio), Giacomo Pirazzoli (UnifI) e Roberta Portas (PUC-Rio) com mediação de Ricardo Artur Pereira Carvalho (ESDI), mas somente os dois primeiros puderam contribuir para esta edição.

No atual cenário, designers têm se deparado com a crescente complexidade constituinte do mundo contemporâneo. A apreensão desta 
complexidade demanda sempre mais cartografias dentre muitas formas não apenas de verificar como também de produzir conexões. À luz de uma sociologia das associações, também conhecida como teoria do ator-rede, designers têm sido estimulados a dirigir um olhar mais atento às 'questões de interesse' do que às 'questões de fato', à ativar um pensamento mais tentacular, reconfigurando a tradicional dimensão do projeto como um processo de "drawing things together". Caracterizado por um fazer mais aberto, experimental e especulativo, essas práticas cartográficas são capazes de potencializar outras narrativas e uma compreensão mais ampla dos diversos agenciamentos que compõem uma determinada questão. Esses pensamentos e práticas reticulares que configuram ao mesmo tempo em que são configurados por visualizações e visualidades estão, por sua vez, intrinsecamente conectados com as novas tecnologias. Podem todas essas redes - de coisas e de representações das coisas - transformar extensa e intensivamente o modo de agir, participar e estar juntos nos espaços reais e virtuais, além dos "entre" espaços e pessoas, e assim constituir uma democracia ampliada e renovada? Desta mesa participaram Clorisval Pereira Jr. (PPG Design-PUC-Rio), Carolina Correia dos Santos (PACC/UFRJ) Frederico Guilherme Bandeira de Araujo, Heitor Levy Ferreira Praça e Letícia Castilhos Coelho (GPMC/IPPUR-UFRJ) com mediação de Daniel Portugal (ESDI).

Também foram abordadas no seminário Design.Com as expansões do design na cidade e a possibilidade de "comuns" urbanos". A cidade contemporânea tem se apresentado como um território fértil para a expansão das fronteiras de atuação do designer, que ora converge ora diverge das práticas e valores de arquitetos, urbanistas e outros profissionais atuantes no território urbano. Esses encontros e desencontros produtivos podem se expressar e até mesmo se enfrentar em termos de escala, de perspectiva (de cima para baixo ou, inversamente, de baixo para cima) ou, ainda, em termos políticos. Com efeito, novas práticas emergem com diversas denominações possíveis - intervenções temporárias, arquiteturas efêmeras, urbanismo tático, microplanejamento criativo - e envolvem a participação de outros atores para além do Estado e das empresas, um amplo leque de "parceiros" com seus próprios saberes, fazeres e poderes. O direito à cidade se reconfigura em ações, com ou sem êxito, pelo comum na/da cidade. Entre formalidade e informalidade, entre criações e capturas, esvaziamentos e engajamentos, que desafios nos traz o design.com? Participaram desta mesa Joy Till (PUC-Rio), Pedro Caetano Eboli (PUC-Rio) e Pedro Claudio Cunca Bocayuva Cunha (NEPP-DH/UFRJ) 
com mediação de Ana Beatriz da Rocha (ESDI). Joy Till não pôde contribuir mas a esta seção foram acrescentados dois artigos: um de Mariana Costard (PPDEsdi/UERJ) e um outro de Barbara Szaniecki, Liana Ventura, Philippe Anastassakis e Talita Tibola.

Todas essas articulações, expansões e conexões envolvem um número sempre maior de instituições, movimentos e atores. As atividades se inserem no Programa de Pós-Graduação da Esdi/UERJ e participam domovimento \#UERJresiste \#EsdiAberta\#DesignEmMovimento

Curadoria e organização do Seminário Design.Com:

Barbara Szaniecki, Camille Moraes, Flavia Secioso, Liana Ventura, Ligia Medeiros, Mariana Costard, Talita Tibola e Zoy Anastassakis.

Organização e edição deste número especial da revista Arcos Design: Barbara Szaniecki, Camille Moraes, Flavia Secioso, Liana Ventura, Mariana Costard e Talita Tibola. 